\title{
Anatomy of a Bathtub Vortex
}

Andersen, Anders Peter; Bohr, Tomas; Stenum, Bjarne; Juul Rasmussen, Jens; Lautrup, B.

Published in:

Physical Review Letters

Link to article, DOI:

10.1103/PhysRevLett.91.104502

Publication date:

2003

Document Version

Publisher's PDF, also known as Version of record

Link back to DTU Orbit

Citation (APA):

Andersen, A. P., Bohr, T., Stenum, B., Juul Rasmussen, J., \& Lautrup, B. (2003). Anatomy of a Bathtub Vortex. Physical Review Letters, 91(10), 104502. https://doi.org/10.1103/PhysRevLett.91.104502

\section{General rights}

Copyright and moral rights for the publications made accessible in the public portal are retained by the authors and/or other copyright owners and it is a condition of accessing publications that users recognise and abide by the legal requirements associated with these rights.

- Users may download and print one copy of any publication from the public portal for the purpose of private study or research.

- You may not further distribute the material or use it for any profit-making activity or commercial gain

- You may freely distribute the URL identifying the publication in the public portal

If you believe that this document breaches copyright please contact us providing details, and we will remove access to the work immediately and investigate your claim 


\title{
Anatomy of a Bathtub Vortex
}

\author{
A. Andersen, ${ }^{1,2, *}$ T. Bohr, ${ }^{1}$ B. Stenum, ${ }^{2}$ J. Juul Rasmussen, ${ }^{2}$ and B. Lautrup ${ }^{3}$ \\ ${ }^{1}$ The Technical University of Denmark, Department of Physics, DK-2800 Kgs. Lyngby, Denmark \\ ${ }^{2}$ Ris $\phi$ National Laboratory, Optics and Fluid Dynamics Department, DK-4000 Roskilde, Denmark \\ ${ }^{3}$ The Niels Bohr Institute, Blegdamsvej 17, DK-2100 Copenhagen Ø, Denmark
}

(Received 11 March 2003; published 5 September 2003)

\begin{abstract}
We present experiments and theory for the "bathtub vortex," which forms when a fluid drains out of a rotating cylindrical container through a small drain hole. The fast down-flow is found to be confined to a narrow and rapidly rotating "drainpipe" from the free surface down to the drain hole. Surrounding this drainpipe is a region with slow upward flow generated by the Ekman layer at the bottom of the container. This flow structure leads us to a theoretical model similar to one obtained earlier by Lundgren [J. Fluid Mech. 155, 381 (1985)], but here including surface tension and Ekman upwelling, comparing favorably with our measurements. At the tip of the needlelike surface depression, we observe a bubble-forming instability at high rotation rates.
\end{abstract}

PACS numbers: 47.32.-y, 47.45.Gx

The generation of strongly localized vorticity is a fascinating and complicating ingredient of a broad variety of fluid flows ranging from vortex shedding at solid surfaces (such as paddles, sand ripples, or insect wings) over flows through turbines to large-scale tornadoes [1]. In general, these flows are poorly understood, since the interplay between fast axial motion and intense, localized vorticity leads to difficult mathematical problems outside the comfortable realm of classical subjects such as potential flow or standard boundary layer theory.

One of the most well-known examples of such flows is the so-called "bathtub vortex," which forms when water drains out of a container. The strong, localized deformation of the free surface makes the vortex beautifully visible, and has made the bathtub vortex the prototype "vortex." This popularity is in stark contrast to the attention which the phenomenon receives in the literature. The few classic papers about it either neglect the axial flow [2] or consider the problem without a free surface [3]. Similarly, textbooks very seldom mention the bathtub vortex, and if they do [4,5] the flow is modeled within potential theory (with the inclusion of an ad hoc viscous core [4]) without incorporating the axial motion. In the vortex core the axial velocity can be high, an essential ingredient of the strong "swirl" [6] which makes the flow so fascinating. Our aim in this Letter is to provide basic understanding of the stationary bathtub vortex: the flow structure, the shape of the free surface, and the interdependence of important characteristics such as the size of the central surface depression, the rate of the out-flow, and the rotation rate in the vortex core.

We study a stationary bathtub vortex in a rotating container with a small drain hole at the bottom. The container is filled to a constant level with water, and the out-flow is driven by gravity. The free surface shape depends strongly on the rate of the out-flow and less on the rotation rate of the container. In the experiment, we fix the size of the drain hole (and thus approximately the flow rate) and vary the rotation rate (angular velocity) $\Omega$. Pictures of the observed free surface profiles are shown in Fig. 1. The surface depression increases with $\Omega$, and at $18 \mathrm{rpm}$ the surface dip has a narrow, needlelike shape and is rotating very rapidly (around $10^{4} \mathrm{rpm}$ ). At higher values of the rotation rate, the tip is no longer stable: Air bubbles detach from it and are dragged down by the surrounding flow as shown in Fig. 2. When $\Omega$ is increased further, the frequency of bubble shedding increases until the air-filled core extends all the way down through the drain hole.

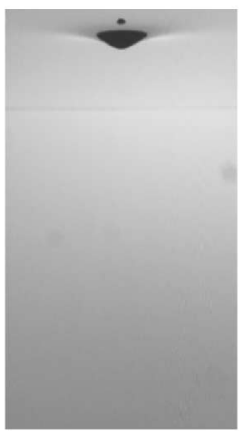

(a)

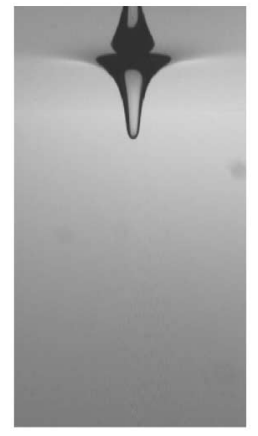

(b)

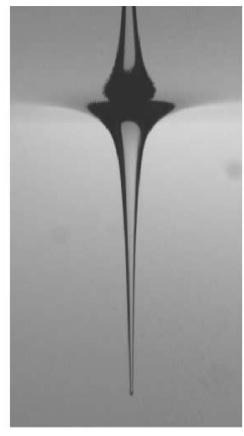

(c)
FIG. 1. The bathtub vortex in a cylindrical container with a drain hole at the center of the bottom (not shown). The fluid in the central region is rotating rapidly, and the depth of the free surface dip depends on the rotation rate of the container. Picture (a) was taken at $6 \mathrm{rpm}$, (b) at $12 \mathrm{rpm}$, and (c) at $18 \mathrm{rpm}$. The drain hole radius was $0.1 \mathrm{~cm}$ and the unperturbed fluid depth $10.9 \mathrm{~cm}$. The rates of the out-flow were (a) $3.62 \mathrm{~cm}^{3} \mathrm{~s}^{-1}$, (b) $3.54 \mathrm{~cm}^{3} \mathrm{~s}^{-1}$, and (c) $3.16 \mathrm{~cm}^{3} \mathrm{~s}^{-1}$. The photographs were taken through the square reservoir tank surrounding the cylindrical tank, and in each photograph the area is $4.0 \mathrm{~cm}$ (horizontally) $\times 7.2 \mathrm{~cm}$ (vertically) and the lower edge of the frame is $4.2 \mathrm{~cm}$ above the drain hole. The structure above the surface is due to reflections. The water is recirculated through a rotationally symmetric inlet at the bottom of the outer cylindrical boundary. 


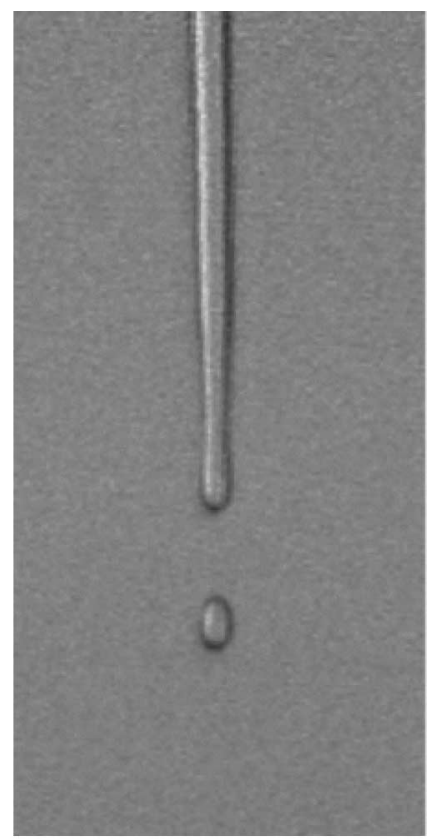

FIG. 2. The tip of the surface depression is unstable when the rotation rate of the container is high. An air bubble has just detached from the tip and is dragged down the drain by the surrounding flow.

The flow structure is surprisingly complex. Figure 3(a) shows an overlay of two flow visualizations made by adding fluorescent dye at the surface (red) and at the bottom inlet (green), respectively. The control parameters correspond to Fig. 1(b). The central down-flow region (red) below the free surface dip is strongly localized, and the downward velocities are of the order of $1 \mathrm{~m} \mathrm{~s}^{-1}$. This region appears similar to a weakly conical drainpipe with a radius comparable to that of the drain hole. If this corresponds to the "esophagus" of the vortex, the "mouth" would appear to be the surface region above the drain, but in the stationary flow only a small fraction of the flow rate through the drain hole actually comes from this region. Nearly all of the flow rate is provided by the thin Ekman layer at the bottom of the container. In this boundary layer, the fluid spirals inward. Close to the drain hole, a large part of it is diverted upward and the rest goes directly down the drain. We note that a similar up-flow was observed by Lewellen [3] close to the drain hole in a rotating flow without a free surface. Figure 3(b) summarizes the flow structure of the bathtub vortex: The flow $1 \mathrm{~cm}$ away from the axis is upward (with velocities of the order of $1 \mathrm{~cm} \mathrm{~s}^{-1}$ ), and most of the fluid passing out through the drain hole has thus taken a contorted path in through the bottom Ekman layer, up through the nextto-central upwelling region, and finally down through the rapidly rotating "porous" central drainpipe.

In the bulk of the container the flow is essentially two dimensional and well described as a central line vortex superimposed on the rigid rotation of the container. This means that the radial velocity $u$ and the vertical velocity $w$ both vanish, whereas the azimuthal velocity
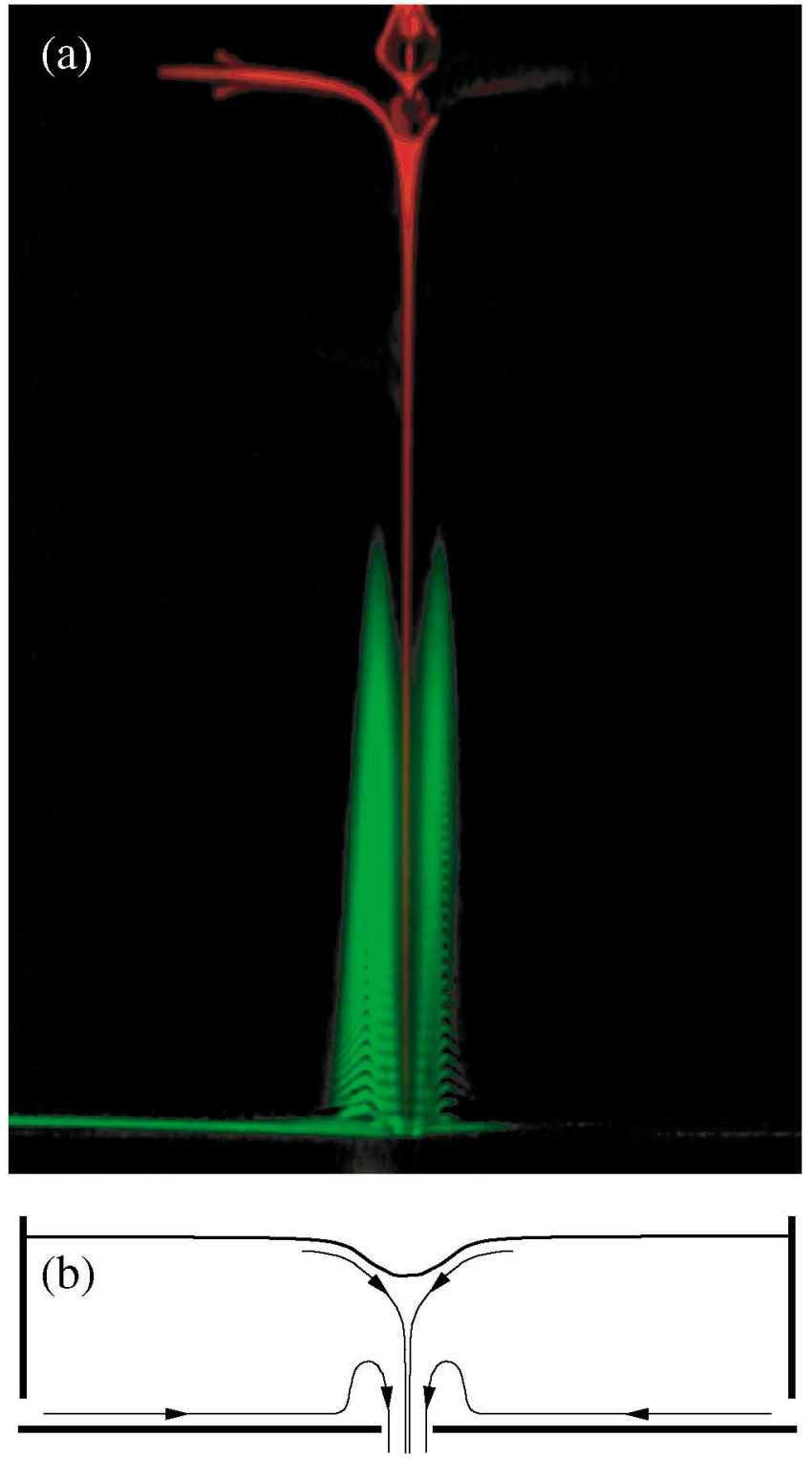

FIG. 3 (color). (a) An overlay of two flow visualizations made by adding fluorescent dye at the surface (red) and at the bottom inlet (green), respectively. The red dye is flowing in a thin surface layer to the central vortex core where it is diverted downwards to the drain hole. The free surface dip is seen above the central red region (the red pattern above the surface is due to reflections). The green dye spirals inward in the thin Ekman layer at the bottom, then upwards around the central drainpipe, and finally down the drain. Only a thin vertical cross section through the axis of symmetry is illuminated and thereby the spiraling upward motion gives rise to the layered structure of the green dye. (b) Sketch of the flow structure (not to scale).

$v=v_{B}(r)=\Omega r+\Gamma / r$, where $\Gamma$ is the line vortex strength. Large deviations from this are observed only near the boundaries and in the central region. Because of the mismatch between the rigidly rotating bottom and the $\Gamma / r$ term in $v_{B}$, an Ekman boundary layer is formed, with the thickness $\delta=\sqrt{\nu / \Omega} \approx 1 \mathrm{~mm}$, where $\nu$ is the kinematic viscosity. By standard linear theory [7], one finds, 
in the reference frame rotating with rotation rate $\Omega$, that $\tilde{u}(r, z)=-(\Gamma / r) \exp (-z / \delta) \sin (z / \delta)$ and $\tilde{\boldsymbol{v}}(r, z)=$ $(\Gamma / r)[1-\exp (-z / \delta) \cos (z / \delta)]$. If we assume that the main flow rate $F$ through the drain hole is carried by the Ekman layer, we get $F \approx-\int_{0}^{\infty} 2 \pi r \tilde{u} d z=\pi \Gamma \delta$, and we can thus express the strength of the line vortex in terms of the flow rate and the rotation rate as $\Gamma \approx$ $F \sqrt{\Omega / \nu} / \pi$. This result has been confirmed experimentally for a source-sink flow without a free surface [8], and our particle tracking measurements at 6,12 , and $18 \mathrm{rpm}$ agree with the prediction to within 10\% [9]. The up-flow is similar to Ekman pumping, which in the linear approximation [7] is proportional to the vertical component of the vorticity, and it vanishes in the bulk since the bulk flow $\Gamma / r$ is irrotational. At the smallest radii $\tilde{v}$ is linear in $r$, corresponding to the approximately rigid rotation of the core. A corresponding up-flow appears due to Ekman pumping, but in this region the nonlinear terms become important and invalidate the linear Ekman theory. In a nonlinear theory $[10,11]$ of the Ekman layer, it is predicted that the boundary layer thickness decreases toward the center and that the up-flow in a vortex core in rigid rotation is reduced due to the nonlinear corrections.

The vertical down-flow in the central core is surprisingly localized. We have thus modeled the flow below the surface dip as a "drainpipe," i.e., as if it was limited by a boundary $r_{0}(z)$ on which $w$ vanishes while $u, v$, and $p$ are matched to the $z$-independent exterior bulk flow. In the vortex core $u \ll w$ and $v \approx w$, and in this region the derivatives with respect to $z$ in the viscous terms can be neglected in a boundary layer approximation of the Navier-Stokes equations. Within the drainpipe, we assumed a parabolic down-flow profile, and we expanded the velocity components and the pressure in powers of $r$. We did not make any assumptions about the functional dependence on $z$, and to lowest order in $r$ the problem reduced to a closed set of differential equations for three functions of $z$. The details are reported elsewhere [9], but the main property used in the following is that the high azimuthal velocity in the vortex core together with the matching to a $z$-independent exterior flow is sufficient to force the flow in the vortex core to have a simple functional dependence where $w$ is approximately linear in $z$ inside the drainpipe, while $r_{0}$ and the central rotation rate, $\Omega_{C}$, are approximately independent of $z$.

That $u$ and $v$ are approximately independent of $z$ whereas $w$ is linear in $z$ was used by Lundgren [12] to derive a set of differential equations for the free surface $h(r)$. With scales $W$ for the vertical velocity in the vortex core and $H$ for the surface height, Lundgren showed that the velocity field has this functional form if the Rossby number $R o \equiv W /(H \Omega) \ll 1$ and if $W^{2} \ll 2 g H$, where $g$ is the gravitational acceleration. These assumptions are valid only for low flow rates and mild depressions of the free surface. In our experiment $R o \approx 10$, but we have a small local Rossby number $R o_{C} \equiv W /\left(H \Omega_{C}\right) \approx 10^{-2}$, defined in terms of the central rotation rate, and this is the relevant number if we restrict our attention to the central drainpipe. Since the out-flow is driven by gravity, our vertical velocities are of the order of the free fall velocity $W \approx \sqrt{2 g H} \approx 1 \mathrm{~m} \mathrm{~s}^{-1}$, and we would thus expect significant corrections to Lundgren's equations which assume hydrostatic pressure. However, as we shall see below, Lundgren's equations lead to surprisingly accurate predictions. The reason is probably that the characteristic vertical velocities near the free surface are considerably smaller than the free fall velocity at the drain hole, due to the velocity increase down the drainpipe and the stagnation point at the tip of the surface depression.

In the following, we apply Lundgren's model [see Ref. [12] equations (4.1), (4.15), and (4.18)] and extend it to include surface tension. Knowledge of the vertical velocity immediately above the Ekman layer $w(z=0)$ makes it possible to compute the product $h u$, since the kinematic boundary condition at the free surface and the incompressibility of the fluid lead to the equation

$$
\frac{1}{r} \frac{d(r h u)}{d r}=w(z=0) .
$$

Inside the down-flow region, we assume a downward plug flow, and in the up-flow region our measurements show an exponential profile:

$$
w(z=0)= \begin{cases}-\frac{Q}{\pi R^{2}} & \text { if } r \leq R \\ \frac{Q e^{-(r-R) / d}}{2 \pi d(R+d)} & \text { if } r>R,\end{cases}
$$

where the flow rate $Q$ for the Ekman upwelling and the length scales $R$ and $d$ are determined experimentally. The flow rate $Q$ is about $50 \%$ of the total flow rate, $F$, since some of the fluid carried by the Ekman layer flows directly down the drain hole. The radius of the downflow region above the drain hole decreases close to the outlet due to the direct outflow from the Ekman layer through the drain hole. The effective value of $R$ is therefore slightly larger than the radius of the drain hole. The radial and the axial Navier-Stokes equations lead with the assumptions $R o_{C} \ll 1$ and $W^{2} \ll 2 g H$ to

$$
\frac{v^{2}}{r}=g \frac{d h}{d r}-\frac{\alpha}{\rho} \frac{d \kappa}{d r}
$$

where $\alpha$ is the surface tension of the air-water interface, $\rho$ is the density of water, and $\kappa=h^{\prime} /\left\{r\left[1+\left(h^{\prime}\right)^{2}\right]^{1 / 2}\right\}+$ $h^{\prime \prime} /\left[1+\left(h^{\prime}\right)^{2}\right]^{3 / 2}$ is the curvature of the free surface. Compared to Lundgren's original equations, we here introduce the surface tension correction to the pressure since it is important in a quantitative comparison with our measurements. Finally, the azimuthal Navier-Stokes equation reduces to the equation

$$
u\left(\frac{d v}{d r}+\frac{v}{r}\right)=\nu\left(\frac{d^{2} v}{d r^{2}}+\frac{1}{r} \frac{d v}{d r}-\frac{v}{r^{2}}\right) .
$$

The problem is thus reduced to solving Eqs. (3) and (4) for $h$ and $v$ using Eq. (1) and the bottom vertical velocity profile in Eq. (2) to eliminate $u$. 

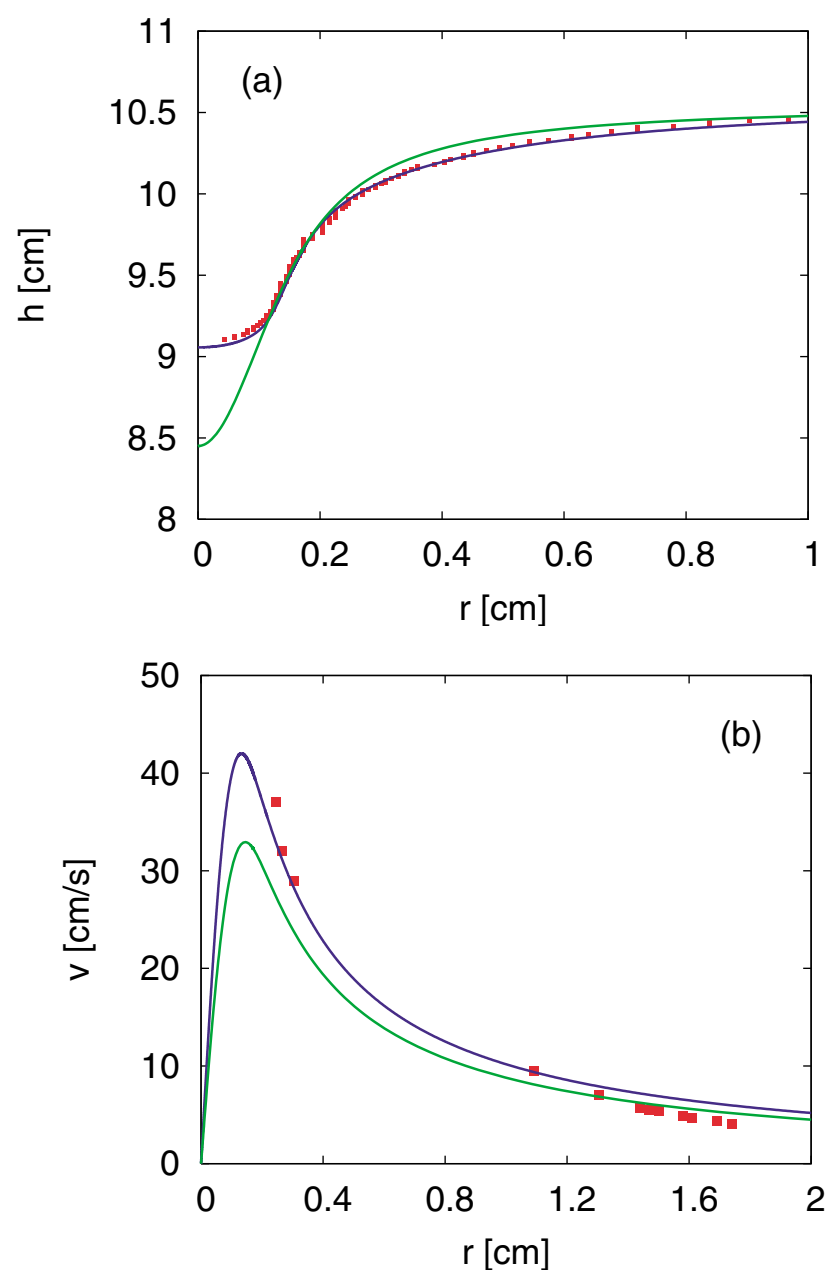

FIG. 4 (color). Numerical solution of Lundgren's equations with surface tension (blue) and without surface tension (green) compared with experimental results (red) at $12 \mathrm{rpm}$. (a) The free surface and (b) the azimuthal velocity in the corotating reference frame. We used $g=981 \mathrm{~cm} \mathrm{~s}^{-2}, \rho=1.0 \mathrm{~g} \mathrm{~cm}^{-3}$, $\nu=0.0089 \mathrm{~cm}^{2} \mathrm{~s}^{-1}, \alpha=65 \mathrm{~g} \mathrm{~s}^{-2}$, and $d=0.35 \mathrm{~cm}$. To obtain the best possible agreement with the measurements, we set $R=0.16 \mathrm{~cm}$ and $Q=1.79 \mathrm{~cm}^{3} \mathrm{~s}^{-1}$ in the solution with surface tension, and $R=0.17 \mathrm{~cm}$ and $Q=1.76 \mathrm{~cm}^{3} \mathrm{~s}^{-1}$ in the solution without surface tension. We measured $\alpha$ in the experiment, and the values of $R, d$, and $Q$ are based on the best fit to our measured vertical velocities $1 \mathrm{~cm}$ above the bottom.

Figure 4 shows a comparison of the measured surface profile and azimuthal velocities at $12 \mathrm{rpm}$ [see Figs. 1(b) and 3(a)] and the corresponding numerical solution of Lundgren's equations with and without surface tension. The numerical solution with surface tension agrees well with the measurements, whereas the model without surface tension overestimates the depth of the central surface depression by $70 \%$. Difficulties in obtaining reliable experimental data for the velocity field and numerical instabilities encountered with Eqs. (3) and (4) at high rotation rates have kept us from making a similar comparison at $18 \mathrm{rpm}$.

The bubble-forming instability (Fig. 2) is induced by surface tension. The capillary instability of a fluid col- umn [13] does not change qualitatively when it is rotating [14]. We therefore speculate that the critical value of $\Omega$ where the instability sets in occurs when the downward drag becomes large enough to overcome the upward buoyancy force and drag the bubbles formed by the capillary instability along with the flow. Using values for the drag on a spherical bubble at high Reynolds number [15], we estimate the critical value of the vertical velocity as $W_{C} \approx g a^{2} /(9 \nu)$, where $a$ is the radius of the bubble. With a bubble radius of $a=0.5 \mathrm{~mm}$, we find $W_{C} \approx$ $25 \mathrm{~cm} \mathrm{~s}^{-1}$, which is in the measured range of $w$. We do not, however, have measurements of $w$ immediately below the surface tip for $\Omega$ around $22 \mathrm{rpm}$ when the tip becomes unstable, and a precise test of the criterion for the tip instability remains to be carried out.

We thank Jens Eggers and Vachtang Putkaradze for drawing our attention to Ref. [12] and Tom Lundgren for helpful suggestions. We are thankful to Morten Ernebjerg for help with measurements of the free surface depression, and to Mark Schram Christensen, Morten Nørgaard Nielsen, and Jacob Richter for inspiration through a series of preparatory experiments. We acknowledge support from the Danish Natural Science Research Council Grant No. 24-56880.

*Present address: Cornell University, Dept. of Theoretical and Applied Mech., Ithaca, NY 14853, USA.

[1] H. J. Lugt, Vortex Flow in Nature and Technology (Krieger, Malabar, Florida, 1995).

[2] H. A. Einstein and H. Li, in Proceedings of the Heat Transfer and Fluid Mechanics Institute, Stanford University, 1951, p. 33.

[3] W. S. Lewellen, J. Fluid Mech. 14, 420 (1962).

[4] H. Lamb, Hydrodynamics (Cambridge University Press, Cambridge, U.K., 1932).

[5] R. P. Feynman, R. B. Leighton, and M. Sands, The Feynman Lectures on Physics (Addison-Wesley, Reading, 1964).

[6] G. K. Batchelor, An Introduction to Fluid Dynamics (Cambridge University Press, Cambridge, U.K., 1967).

[7] D. J. Acheson, Elementary Fluid Dynamics (Oxford University Press, Oxford, 1990).

[8] M. Mory and N. Yurchenko, Eur. J. Mech. B 12, 729 (1993).

[9] A. Andersen, Ph.D. thesis, The Technical University of Denmark, 2002).

[10] J. M. Owen, J. R. Pincombe, and R. H. Rogers, J. Fluid Mech. 155, 233 (1985).

[11] A. Andersen, B. Lautrup, and T. Bohr, J. Fluid Mech. 487, 81 (2003).

[12] T. S. Lundgren, J. Fluid Mech. 155, 381 (1985).

[13] P. G. Drazin and W.H. Reid, Hydrodynamic Stability (Cambridge University Press, Cambridge, U.K., 1981).

[14] L. M. Hocking, Mathematika 7, 1 (1960).

[15] J. Magnaudet and I. Eames, Annu. Rev. Fluid Mech. 32, 659 (2000). 